\title{
Multi-station Feeding Robot Structure Design
}

\author{
Lin Hong $^{\mathrm{a}}$, Yu Sun ${ }^{\mathrm{b}}$ and Chunping Cao \\ School of Mechanical Engineering, Nanjing University of Science and Technology, Nanjing 210094, \\ China. \\ ${ }^{a}$ Corresponding author: 15105171448@163.com \\ bsunyu@njust.edu.cn, ${ }^{c} c c p \_a l i c e @ 163 . c o m$
}

Keywords: multi-station precision hot forging; feeding robot; rack and pinion; double rail slider

Abstract: In order to realize the automatic production of hot die forging, a matching feeding manipulator is designed for the core equipment of the hot forging production linemulti-station hot die forging press. The manipulator designed by this project adopts the rack and pinion transmission mode and adopts the double rail slider positioning method. Finally, the operating parameters of the manipulator are measured in practical applications, and the experimental results prove that the manipulator can meet the production requirements.

\section{Introduction}

The forging process is the main production method for manufacturing key parts and transmission parts of automobiles, and it has a very important position in automobile forging [1-2]. With the rapid development of automobile manufacturing technology, the competitive pressure of enterprises is increasing. High precision, high performance and low cost have become the core ways for enterprises to enhance their competitiveness. The traditional single-machine forging method can not meet the modern forging demand [3-4]. Multi-station precision hot forging is one of the modern precision manufacturing technologies and has been widely used at home and abroad [5]. Multistation hot die forging technology has changed the previous single-machine connection production method, which has high production efficiency, reduced production cycle and improved production efficiency. It is especially suitable for mass production of auto parts, such as gear blanks and methods for automobile transmissions. At present, multi-station hot forging process has become an indispensable part of mechanical manufacturing [6].

In this paper, the mechanical structure of the multi-station feeding manipulator is designed from the application requirements of the multi-station feeding robot, and the acceleration test of the manipulator is carried out.

\section{Three-dimensional modeling of multi-station hot die forging robot}

The multi-station feeding manipulator designed by this subject is used for automatic hot forging production line. The hot forging process has higher requirements on the matching precision of hot forging press and multi-station feeding manipulator, so it is necessary to ensure that there is no 
interference occurs during exercise. in the structural design.

The multi-station feeding robot $\mathrm{X}, \mathrm{Y}, \mathrm{Z}$ three axes are positioned by a Cartesian coordinate system, which simplifies the mechanical structure and kinematics modeling while ensuring the transmission accuracy. Through the linear drive module, the three functions of clamping-opening, lifting-lowering, and transferring-retracting are realized. The three actions are driven by the threephase asynchronous motor to ensure high speed and high precision

The Z-axis drive structure includes a driver, a drive mounting plate, a double guide rail, a double slide, a drive rack, a drive arm, a sensor, a proximity switch, and a mounting plate. The drive includes a drive motor, a coupling, a reducer, a transmission gear, and an automatic oiler. The driving rack and the double rail are mounted on the mounting board 2, and the transmission gear and the double slider are mounted on the mounting board 1, and the mounting board 2 is integrally mounted with the rack. The Z-axis drive shaft is connected to the left and right drive arms, and the middle is connected by a coupling to ensure the synchronization of the movement at both ends.

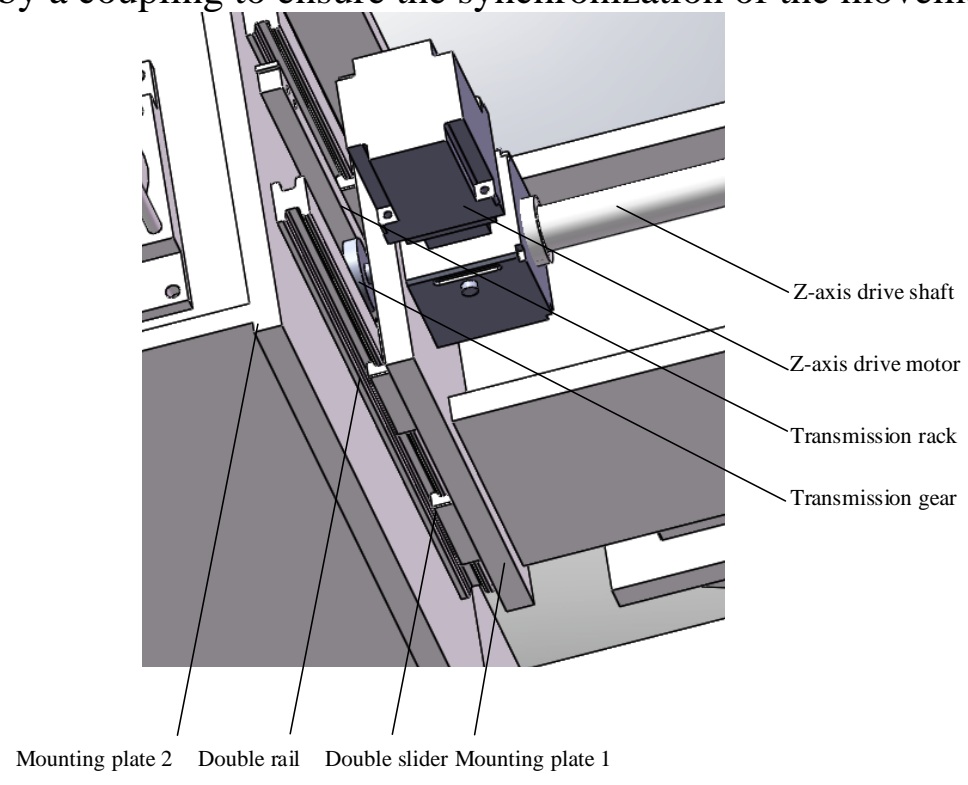

Figure 1. Z-axis drive

The fixed end of the balance cylinder is connected to the support arm of the z-axis drive structure. The balance cylinder mounting structure is as shown in the figure below. The balance cylinder can drive the z-axis support arm upward to balance the weight of the Y-axis drive structure and the Xaxis drive structure and the connecting arms and clamps.

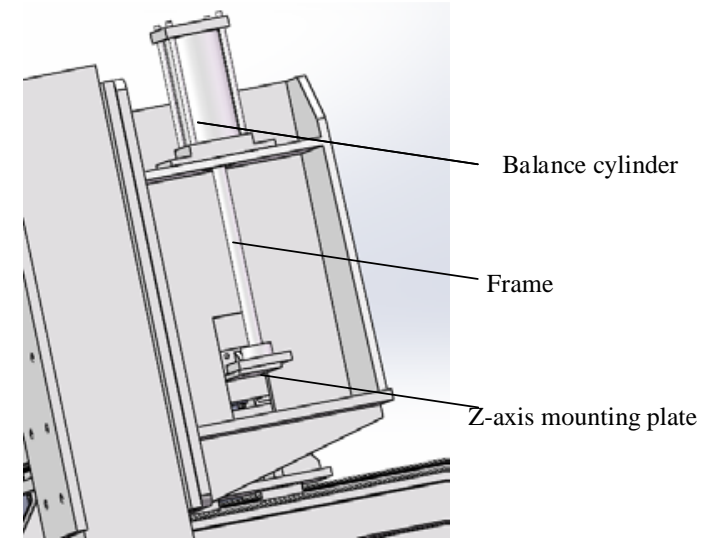

Figure 2. Balance cylinder 
The figure below shows the balanced cylinder and Z-axis drive structure.

The $\mathrm{X}$-axis drive structure consists of a driver, a drive mounting plate, a double guide rail, a double slide, a drive rack, a sensor, a proximity switch, and a mounting plate. The drive includes a drive motor, a coupling, a reducer, a transmission gear, and an automatic oiler. The drive is mounted on the drive mounting plate, the drive rack and the double slide are mounted under the beam, the double rail and the drive gear are mounted on the front of the drive arm, and the double slide is mounted on the drive mounting plate. The automatic oiler passes through the felt gear and The drive racks mesh with each other.

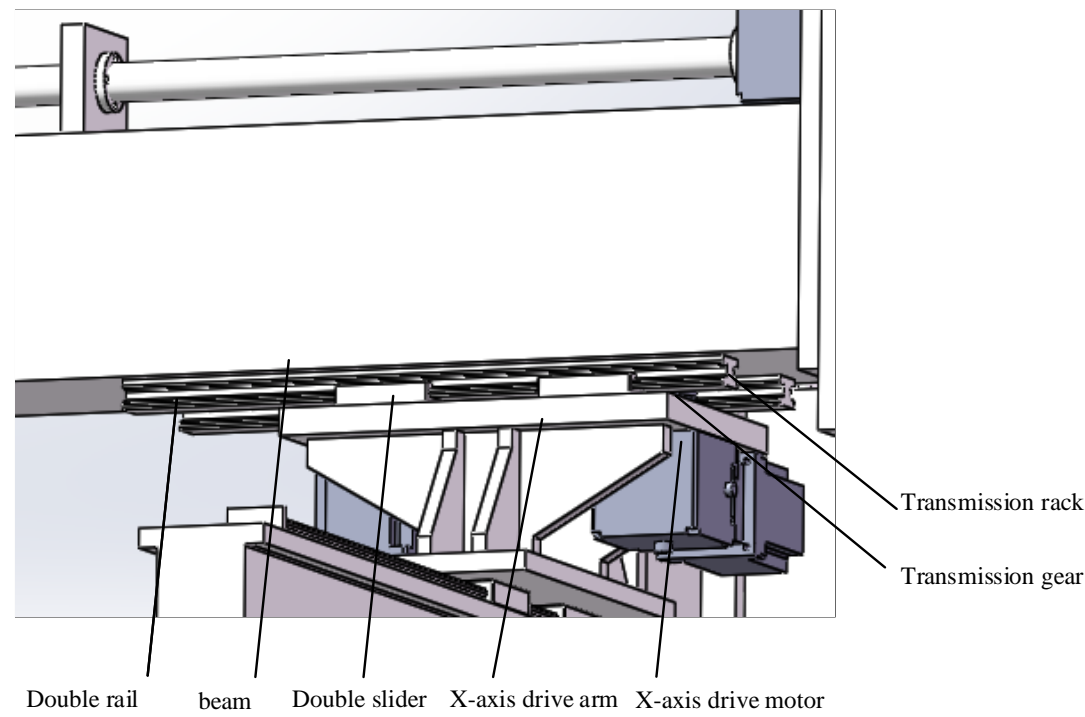

Figure 3. X-axis drive structure

The Y-axis drive structure comprises a driver, a drive mounting plate, a double guide rail, a double slide, a drive rack, a sensor, a proximity switch, and a mounting plate. The drive includes a drive motor, a coupling, a coupling, a reducer, a transmission gear, and an automatic oiler. The driver is mounted on the drive mounting plate, the drive rack is mounted on the side of the drive arm, the double rail and the rack are mounted directly on the drive beam, the double slide is mounted on the X-axis drive arm, and the automatic oiler is driven by the felt gear The racks mesh with each other.

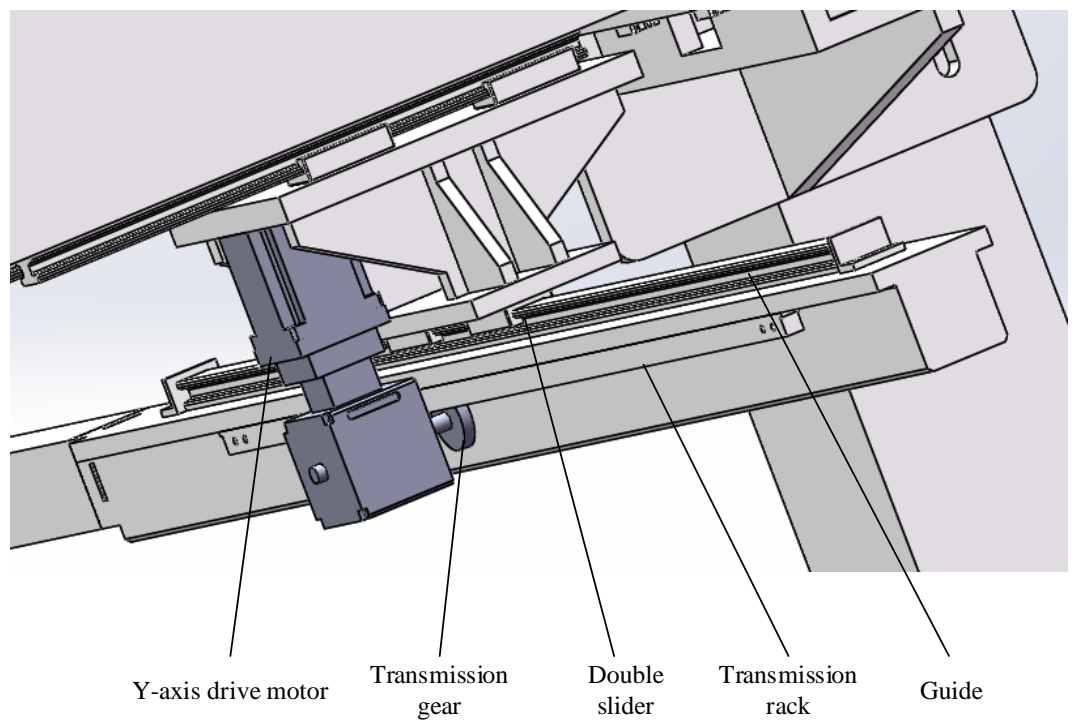

Figure 4. Y-axis drive structure 
The overall structure is obtained by assembling the above structure as shown in Figure 5.

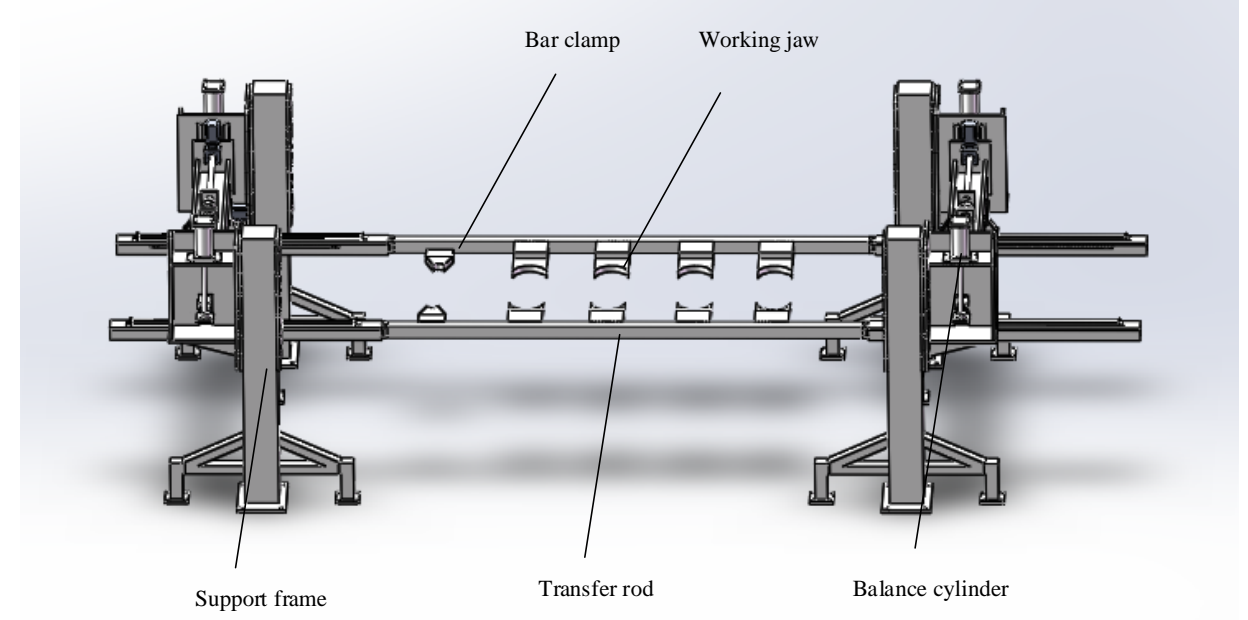

Figure 5. Overall assembly structure

\section{Experiment and analysis}

The acceleration test of feed manipulator arm was studied. Based on the integral calculation, the speed of robot arm can be obtained. Two acceleration sensors (Kistler 8786A5) were attached to the left and the right robot arm. The data collection was implemented in LabVIEW environment. Other experimental equipments include: USB Compact DAQ Chassis (NI cDAQ-9174), input module (NI-9232). The accelerations in three directions (X, Y and Z axis) were tested, respectively. The testing site is shown in Figure 6. The curves of testing speeds are shown in Figure 7.

It can be seen from Figure 6 that the speeds of the left and the right arms are almost equal, the error between them is less than $1 \%$, and the time difference is less than $0.05 \mathrm{~s}$. The two arms have a good synchronization performance when the speed is constant or changing. The system has a good robustness, and can meet the requirement of production.

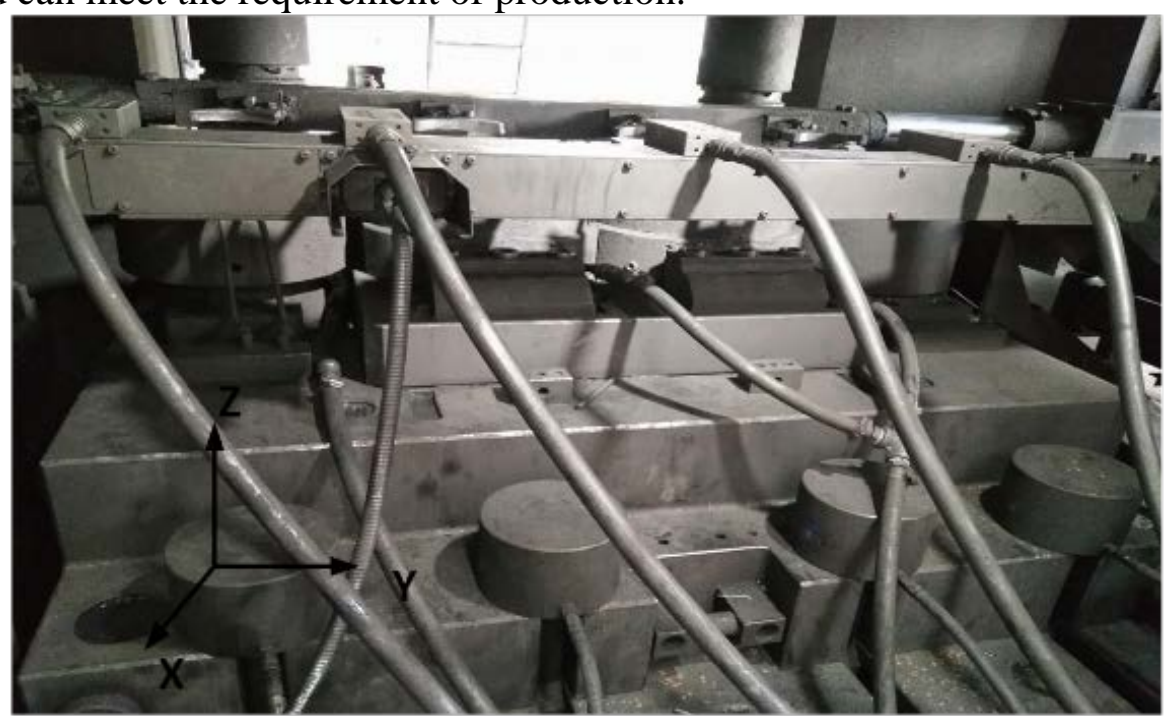

Figure 6. Acceleration testing site 


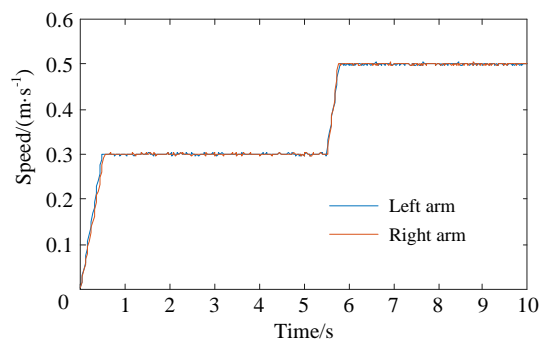

a) X-axis speed

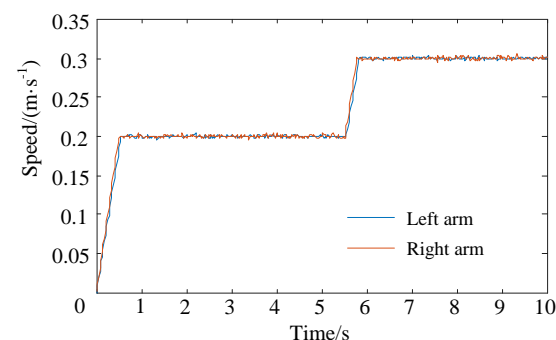

b) Y-axis speed

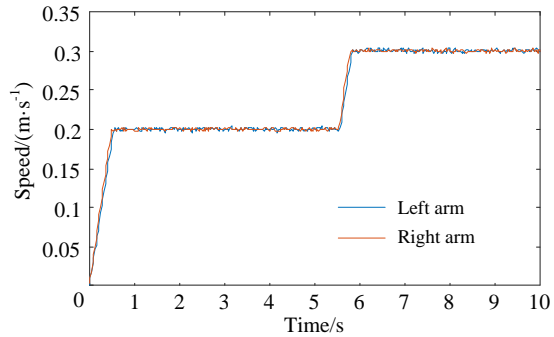

c) Z-axis speed

Figure 7. The curves of speeds

\section{Conclusions}

In this paper, the overall structure design of the multi-station feeding manipulator is completed, and it is applied in actual production and realized the automatic production of hot die forging. The acceleration test experiment is carried out for the designed manipulator. The result shows that the manipulator has good stability and can meet the production demand.

\section{Acknowledgements}

This research is support by The Key Research and Development Project of Jiangsu Province, China (Industrial Forward-Looking and Generic Technology) (BE2015011-3).

\section{References}

[1] J. Peng and S. Yong, Application progress and improvement suggestion of forging automation technology, Metal Processing (thermal processing), (2014), Vol. 21, pp. 6-7.

[2] Z. Huixu, Application analysis of hot dieforging press, Metal processing (thermal processing), (2014), Vol. 5, pp. 54. [3] W. Yannian, and D. Heng, Control system design on automatic feeding manipulator in the multi-station press line, Forging technology, (2016), Vol. 41, No. 06, pp. 55-60.

[4] H. Lin, S. Yu and C. Chunping, Mechanism design and motor synchronous control of multi-station feeding manipulator, Forging \& stamping technology, (2018), Vol. 43, No. 06, pp. 89-93.

[5] C. Hao. and $\mathrm{Yu}, \mathrm{S}$. Development and application of reliability test platform for high-speed punch machine clutch brake system, Journal of Mechanical Science and Technology, (2017), Vol. 31, No. 1, pp. 53-61.

[6] Z. Lin and X. Caixia, Optimization Design of Control System for Hot Die Forging Press Based on EtherCAT, Hot Working Technology, (2018), Vol.47, No.9, pp. 155-158. 